\title{
The effect of a lateral wedge insole and a subtalar strap on gait parameters in knee osteoarthritis
}

\author{
Elham Esfandiari ${ }^{1,2}$, Mojtaba Kamyab*1, Hamid Reza Yazdi ${ }^{3}$, Mohammad Ali Sanjari ${ }^{4}$, \\ Fatemeh Navvab Motlagh ${ }^{5}$
}

Received: 7 Oct 2018

Published: 30 Dec 2019

\begin{abstract}
Background: Lateral wedge insole (LWI) aims to reduce loading on medial compartment of tibiofemoral joint in mild knee osteoarthritis (KOA). This effect may be augmented by concomitant use of subtalar strap to fix the ankle joint. Moreover, longitudinal arch support embedded in insoles can cause foot comfort and may be beneficial for people with KOA. Therefore, this study aimed to assess the immediate effect of LWI with an arch support with and without a subtalar strap on the kinetics and kinematics of walking in mild KOA.
\end{abstract}

Methods: A convenient sample of 17 individuals with mild KOA (Kellgren and Lawrence grade II), aged $\geq 40$ years were assessed in 3 conditions: without the insole; LWI; and LWI with a subtalar strap, where an arch support was embedded in all insoles. The primary outcomes were external knee adduction moment and angular impulse. The secondary outcomes were hip flexion and adduction moments, knee flexion angle, ankle eversion moment, and walking speed. The repeated measurements ANOVA was used to compare the primary and secondary outcomes between the conditions using SPSS. Significance level was set at 0.05.

Results: LWI and a subtalar strap can significantly increase the knee flexion angle at $0 \%-15 \%$ of the stance phase compared to no insole $(\mathrm{p}<0.001)$. No other changes were observed $(\mathrm{p}=0.142)$.

Conclusion: LWI with an arch, with or without a subtalar strap, cannot impose any immediate changes on the kinetics and kinematics of lower limb joints during walking in people with mild KOA.

Keywords: Osteoarthritis, Knee, Orthotics, Gait

Conflicts of Interest: None declared

Funding: Research Committee of Iran University of Medical Sciences

*This work has been published under CC BY-NC-SA 1.0 license.

Copyright $($ Iran University of Medical Sciences

Cite this article as: Esfandiari E, Kamyab M, Yazdi HR, Sanjari MA, Navvab Motlagh F. The effect of a lateral wedge insole and a subtalar strap on gait parameters in knee osteoarthritis. Med J Islam Repub Iran. 2019 (30 Dec);33:157. https://doi.org/10.47176/mjiri.33.157

\section{Introduction}

Knee osteoarthritis (KOA) is the fourth common progressive joint disease that is associated with aging, and some livelihoods such as kneeling and squatting, and heavy physical activities (1), with higher incidence in developing countries. Moreover, the prevalence of KOA is higher in females compared to males (2). The most associated factor in KOA progression is the increased load-

Corresponding author: Dr Mojtaba Kamyab, kamyba.m@iums.ac.ir

1. Department of Orthotics and Prosthetics, School of Rehabilitation Sciences, Iran University of Medical sciences, Tehran, Iran

2. Graduate Program in Rehabilitation Sciences, University of British Columbia, Vancouver, BC, Canada

3. Department of Orthopedic Surgery, School of Medicine, Iran University of Medical Sciences, Tehran, Iran

4. Department of Basic Rehabilitation Sciences, School of Rehabilitation Sciences, Iran University of Medical Sciences, Tehran, Iran

5. Biomedical Engineering School, Amir Kabir University of Technology, Tehran, Iran ing on the medial compartment of the knee joint, which leads to the femorotibial varum. This loading is indirectly measured with external knee adduction moment (EKAM) $(3,4)$. Biomechanical interventions such as knee braces and lateral wedge insoles (LWIs) are prescribed as one of the most common interventions with no adverse effect for $\operatorname{KOA}(5,6)$. LWI aims to reduce EKAM $(7)$, which im-

$\uparrow$ What is "already known" in this topic:

$\mathrm{KOA}$ is associated with the increase in the loading on medial compartment of tibiofemoral joint, which is quantified by EKAM. The augmented effect of LWI and a subtalar strap was shown by the reduction of EKAM in people with KOA.

\section{$\rightarrow$ What this article adds:}

This study showed that LWI and a subtalar strap with embedded arch support cannot affect EKAM, hip, and ankle joint kinetics in people with KOA, except on the knee flexion angle in $0 \%-15 \%$ of the stance phase. 
proves pain and physical function $(8,9)$.

LWI can shift the calcaneus into a valgus position which shifts the center of pressure (COP) toward the lateral compartment of knee joint and may decrease the pain intensity in people with KOA (10). However, movement of talus by using a LWI may decrease the correction of femorotibial varum (11). Therefore, fixing the talus movement with a subtalar strap may increase the valgus angulation of the talus and augment the femorotibial angle change $(11,12)$. Moreover, the effect of LWI can be increased if it is joined with the foot comfort, which is associated with concomitant use of longitudinal arch support $(13,14)$. Although the augmented effect of LWI and a subtalar strap have been shown by reducing EKAM in KOA, the impact of using LWI with an arch support and a subtalar strap in reduction of EKAM has not yet been known $(11,12)$. Slower walking speed (15) and prolonged stance phase (16) are 2 features of gait in individuals with KOA that could be incorporated in EKAM by knee adduction angular impulse (17), which combines the magnitude and duration of EKAM (17).

Moreover, it is not known whether the concurrent use of a subtalar strap and LWI can create any secondary gait changes in lower limb joints (16). Therefore, it was aimed to conduct a pre- and post-intervention study to assess the immediate effect of LWI with an arch support and a subtalar strap on EKAM and knee adduction angular impulse, as the primary outcome measures in individuals with mild KOA (Kellgrene and Lawrence (K/L) grade II) (18). Furthermore, the effect of LWI with an arch support and a subtalar strap was assessed on hip adduction moment, hip flexion moment, knee flexion moment, knee flexion angle, ankle eversion moment, and walking speed, as the secondary outcomes.

\section{Methods}

\section{Study design}

This study was a pre- and post-intervention trial to evaluate the immediate effect of LWI with an arch support and a subtalar strap in patients with mild KOA. The statement checklist of Transparent Reporting of Evaluations with Nonrandomized Design (TREND) was used for protocol design and report (19). Human Research Ethics Committee of Iran University of Medical Sciences approved the study design, and all participants signed written informed consent forms. Also, this study was registered in registry of clinical trials (IRCT 201104185520N4).

\section{Sample size}

Sample size was 17 based on the power of 0.8 , the effect size of 0.25 , and the instrument reliability of 0.7 , which is the minimum requirement for correlation among the residuals for the repeated measures for biomedical engineering instruments (20). The $\mathrm{G}^{*}$ Power package was used to calculate the sample size (21).

A convenient sample of 17 community dwelling adults aged over 40 years with mild KOA were recruited by orthopedic specialist (HRY) referrals. Other inclusion criteria were as follow: grade II of the medial tibiofemoral OA in at least 1 knee, according to the American College of Rheumatology criteria (22) and $\mathrm{K} / \mathrm{L}$ grading system (18) (eg, 15\%-25\% narrowing of the medial compartment joint space) (23); pain above level 3 on the Visual Analogue Scale (VAS); and radiographic osteophyte at the joint space.

Exclusion criteria were as follow: secondary KOA associated with trauma, K/L grade I, III or IV; past knee joint surgery; arthritis at hip or lumbar joints; significant knee ligament laxity; therapeutic injection at knee joint over the past 6 months; nervous system disorders restricted voluntary movement; perception disorder; chronic lower back pain; limb length discrepancy; using orthotic treatments for knee joint or foot (10); any foot problems restricting insole use such as hallux stiffness; knee joint valgus alignment $>185^{\circ}$, as LWIs are unlikely to benefit such individuals; recurvatum or severe varus malalignment; $\mathrm{OA}$ in lateral compartment of the knee; and other problems limiting independent walking. The participants called the evaluator prior to data collection to be screened for their foot size and the involved limb with OA.

\section{Interventions}

The LWIs were prepared from high density ethyl-vinyl acetate (70 Shore A) (24) and covered by leather (Fig. 1). The insole for involved limb had $5^{\circ}$ full-length lateral wedge (25). To avoid leg length discrepancy, a neutral insole was used on the uninvolved side. All insoles had a medial longitudinal arch support (25), and the insoles were fitted in gymnastics shoes to keep in place. A $5 \mathrm{~cm}$ width elastic strap with Velcro was used as subtalar strap for the involved side (11). The subtalar strap was twisted and fixed around the ankle joint in figure of 8 (Fig. 2). All
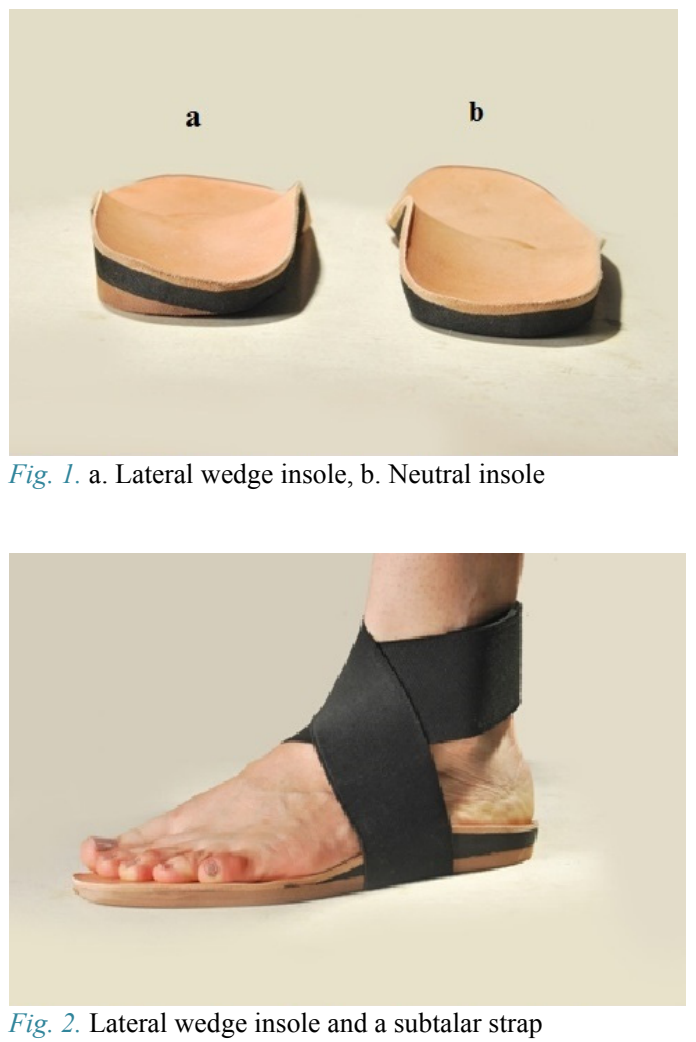
insoles and subtalar straps were prepared before data collection. Three conditions were tested randomly: (a) without insole, (b) with a LWI, and (c) with a LWI and a subtalar strap. Gymnastic shoes were used for all 3 conditions, as the optimal use of a LWI could be in conjunction with socks or flat soft footwear (26), and the concomitant use of heeled footwear could decrease the effect of LWI (26). Each participant walked for 10 minutes before recording the data to get used to LWI and the subtalar strap.

The immediate effect of interventions was examined to consider the mechanical effect of LWI and a subtalar strap (27), as the immediate changes in biomechanical parameters could predict the outcomes following a longer treatment period $(28,29)$.

\section{Gait measurement}

Age, gender, body weight and height of each participants were recorded by an evaluator. To capture the kinetic and kinematic data of lower limb joints, a 6-camera Vicon motion measurement system (640, Oxford Metrics, Oxford, UK), with sampling rate of 100 frames per seconds $(\mathrm{Hz})$, and 2 Kistler force plates (Kistler 9286BA, Switzerland), with sampling rate of $200 \mathrm{~Hz}$, were used. One gait cycle of the involved limb was assessed.

Seven passive reflective markers were placed unilaterally on the skin over the position of the anterior superior iliac spine (ASIS), greater trochanter, lateral condyle of the femur, head of the fibula, lateral malleolus of the fibula, the second metatarsal head, and posterior aspect of the calcaneus. The participants performed an 8-meter level walking at a self-selected walking speed. Three successful walking trials were recorded in each condition. Also, a successful trial was defined as recording of 1 gait cycle of the involved limb. In addition, a 3-minute rest between each trial was offered to participants.

To calculate the external moment of hip, knee, and ankle joints, it was assumed that each segment (thigh, shank, and foot) was a rigid body. The marker positions at the greater trochanter, the lateral condyle of the femur, and the lateral malleolus were used to calculate joint center positions. External moments were calculated for hip, knee, and ankle using the inverse dynamics via MATLAB program (7.0.4.365, R14, version 2.0). The knee flexion angle was calculated from the position of the markers and a coordinate system fixed in tibial as a reference system. The external moments of lower limb joints were normalized with the body weight and height $(\mathrm{Nm} /[\mathrm{BW} * \mathrm{Ht}] \%)$. Also, each trial was normalized to $100 \%$ of a gait cycle and averaged the 3 trials. The following subphases throughout the stance phase were determined: loading as the $0 \%-15 \%$ of the stance phase, early stance as the $15 \%$ $40 \%$ of the stance phase, mid-stance as the $40 \%-60 \%$ of the stance phase, and the late stance as the last $40 \%$ of the stance phase (30). EKAM at the early, mid, and late stance, and the positive knee adduction angular impulse $\left(\mathrm{Nm} . \mathrm{s} /\left[\mathrm{BW}{ }^{*} \mathrm{Ht}\right] \%\right.$ ) (calculated as the positive area under the EKAM-time curve) were used as primary outcome measures. The peak of the hip flexion moment, hip adduction moment, knee flexion moment, and ankle eversion moment at the early, mid, and late stance were used as the secondary outcomes. Moreover, knee flexion angle was determined during loading and early stance phase. The peak of knee extension angle was also determined at midstance. Furthermore, projection of the ASIS marker through the walk way was used to calculate the walking speed $(\mathrm{m} / \mathrm{s})$. Figure 3 represents the graphs of EKAM and knee flexion angle in one gait cycle of a patient in 3 conditions.

\section{Statistical analyses}

SPSS (Release 18 for Windows, 2009, Chicago, SPSS Inc) was used for statistical analyses. Descriptive statistics was reported as mean, standard deviation (SD), and 95\% confidence interval [CI] due to the normality of data, which was assumed by applying an analysis of ShapiroWilk test. Three conditions were compared by applying an analysis of repeated measurement. All $p$ values less than 0.05 were considered statistically significant.

\section{Results}

\section{Participants}

The mean \pm SD age and BMI of the female participants $(n=17)$ were $45.65 \pm 4.83$ years and $30.94 \pm 3.68 \mathrm{~kg} / \mathrm{m}^{2}$, respectively. The average pain measured on VAS score
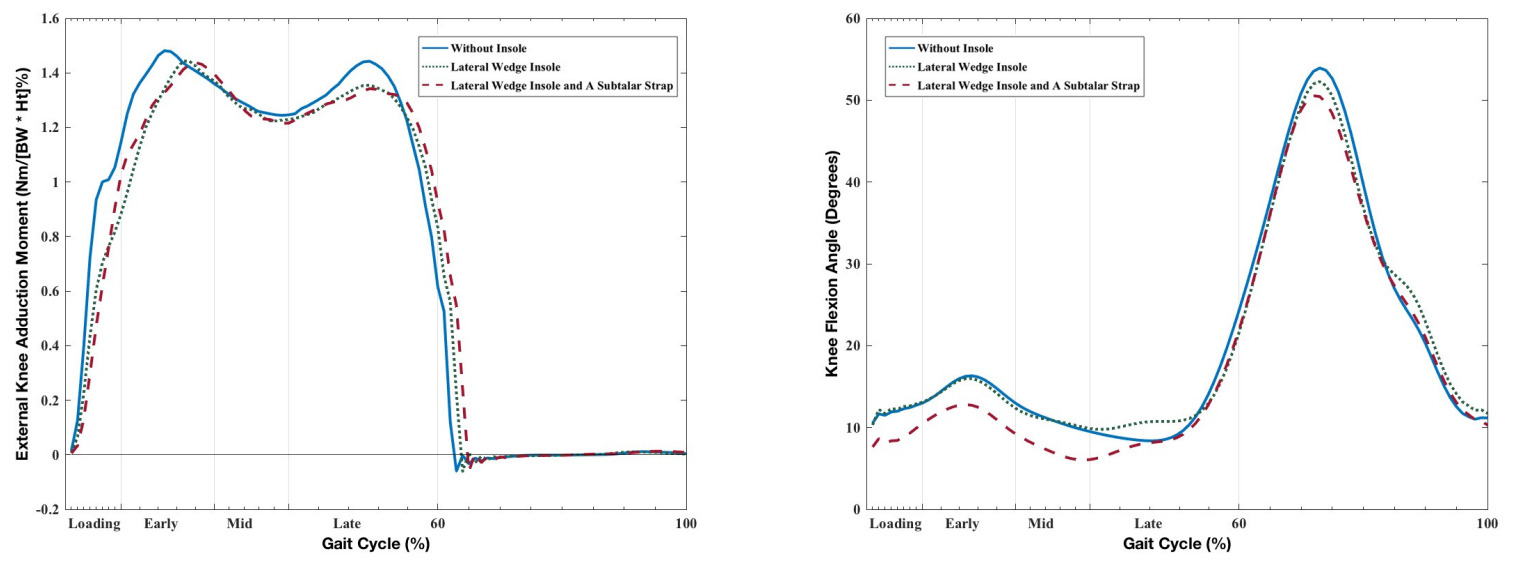

Fig. 3. External knee adduction moment and knee flexion angle in 3 conditions: without insole, lateral wedge insole, and lateral wedge insole and a subtalar strap, at loading, early, mid and late stance phase of a gait cycle for a patient. 
was $5.75 \pm 1.74$. None of the participants complained about the adverse effect of LWI during the tests.

\section{Gait analysis}

No significant difference was observed between 3 conditions (without insole, LWI, and LWI), with a subtalar strap on EKAM at the early, mid, and late stance and the positive knee adduction angular impulse (Nm.s/[BW*Ht]\%), as primary outcome measures (p values $=0.416,0.924,0.907$, and 0.563, respectively) (Fig. 4) (Table 1). The effect of 3 conditions on hip adduction moment was insignificant at the early and late stance phase of gait $(\mathrm{p}=0.498$ and 0.773 , respectively). There was no difference between the 3 conditions on hip flexion moment at early, mid, and late stance phase of gait $(p=0.269,0.497$, and 0.166 , respectively). Moreover, no significant difference was detected between the 3 conditions on knee flexion moment, ankle eversion moment at the early, mid, and late stance phase of gait $(\mathrm{p}=0.148)$ and walking speed $(p=0.142)$. However, a significant difference was found between the knee flexion angle at loading in 3 conditions $(p=0.035)$. In pairwise comparisons, LWI and LWI and a subtalar strap were statistically different $(p=0.001)$. Table 2 demonstrates the descriptive characteristics of outcomes.

\section{Discussion}

The aim of this study was to assess the effect of LWI and a subtalar strap on gait characteristics of patients with mild KOA. The results showed no statistically significant difference between 3 conditions (without insole, LWI, and LWI) and a subtalar strap on EKAM, knee adduction angular impulse, and other kinetic and kinematic variables of lower limb joints and walking speed.

In a systematic review and meta-analysis (7), a more conclusive low to medium effect of LWI on EKAM at early, late stance, and knee adduction angular impulse were reported. However, a minimal effect of LWI with an arch support on the first peak of EKAM at the earlystance and knee adduction angular impulse was reported (7). The results of this study showed no significant effect of LWI, with and without a subtalar strap, on primary and secondary outcomes. According to the published studies, the findings on the effect of LWI with an embedded arch
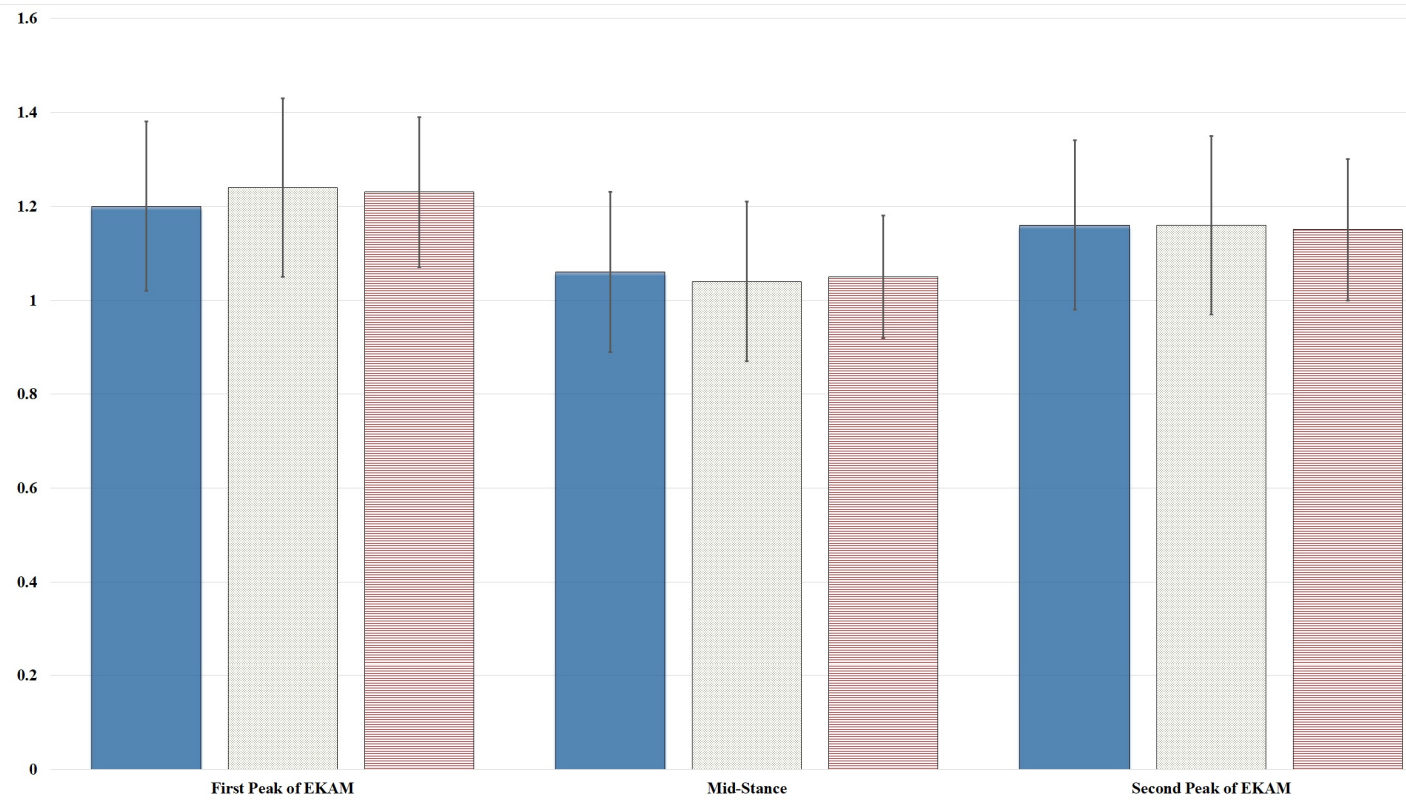

EWithout Insole

Insole Lateral Wedge Insole $\quad$ Lateral Wedge Insole and A Subtalar Stra

Fig. 4. Mean \pm SD of external knee adduction moment at early, mid, and late stance in 3 conditions: without insole, lateral wedge insole, and lateral wedge insole and a subtalar strap $(\mathrm{n}=17)$.

Table 1. Descriptive statistics for the external knee adduction moment and knee adduction angular impulse for patients with mild knee osteoarthritis $(\mathrm{n}=17)$

\begin{tabular}{|c|c|c|c|c|}
\hline \multirow[t]{2}{*}{ Variables $(\mathrm{n}=17)$} & Without insole & Lateral wedge insole & Lateral wedge insole and subtalar strap & \multirow[t]{2}{*}{$\mathrm{p}$} \\
\hline & $\begin{array}{c}\text { Mean } \pm \text { SD } \\
(95 \% \mathrm{CI})^{\#}\end{array}$ & $\begin{array}{l}\text { Mean } \pm \text { SD } \\
(95 \% \mathrm{CI})^{\#}\end{array}$ & $\begin{array}{l}\text { Mean } \pm \text { SD } \\
(95 \% \mathrm{CI})^{\#}\end{array}$ & \\
\hline Early-Stance EKAM ${ }^{*}$ & $1.20 \pm 0.18$ & $1.24 \pm 0.19$ & $1.23 \pm 0.16$ & 0.416 \\
\hline$\left(\mathrm{Nm} /\left[\mathrm{BW}^{*} \mathrm{Ht}\right] \%\right)$ & $(1.12,1.29)$ & $(1.16,1.34)$ & $(1.15,1.30)$ & \\
\hline Mid-Stance EKAM ${ }^{*}$ & $1.06 \pm 0.17$ & $1.04 \pm 0.17$ & $1.05 \pm 0.13$ & 0.924 \\
\hline$(\mathrm{Nm} /[\mathrm{BW} * \mathrm{Ht}] \%)$ & $(0.98,1.14)$ & $(0.96,1.13)$ & $(0.99,1.12)$ & \\
\hline Late-Stance EKAM ${ }^{*}$ & $1.16 \pm 0.18$ & $1.16 \pm 0.19$ & $1.15 \pm 0.15$ & 0.907 \\
\hline$(\mathrm{Nm} /[\mathrm{BW} * \mathrm{Ht}] \%)$ & $(1.08,1.25)$ & $(1.07,1.25)$ & $(1.08,1.22)$ & \\
\hline Knee Adduction Angular Impulse & $0.57 \pm 0.08$ & $0.56 \pm 0.08$ & $0.55 \pm 0.09$ & 0.563 \\
\hline$(\mathrm{Nm} . \mathrm{s} /[\mathrm{BW} * \mathrm{Ht}] \%)$ & $(0.52,0.61)$ & $(0.52,0.61)$ & $(0.51,0.60)$ & \\
\hline
\end{tabular}




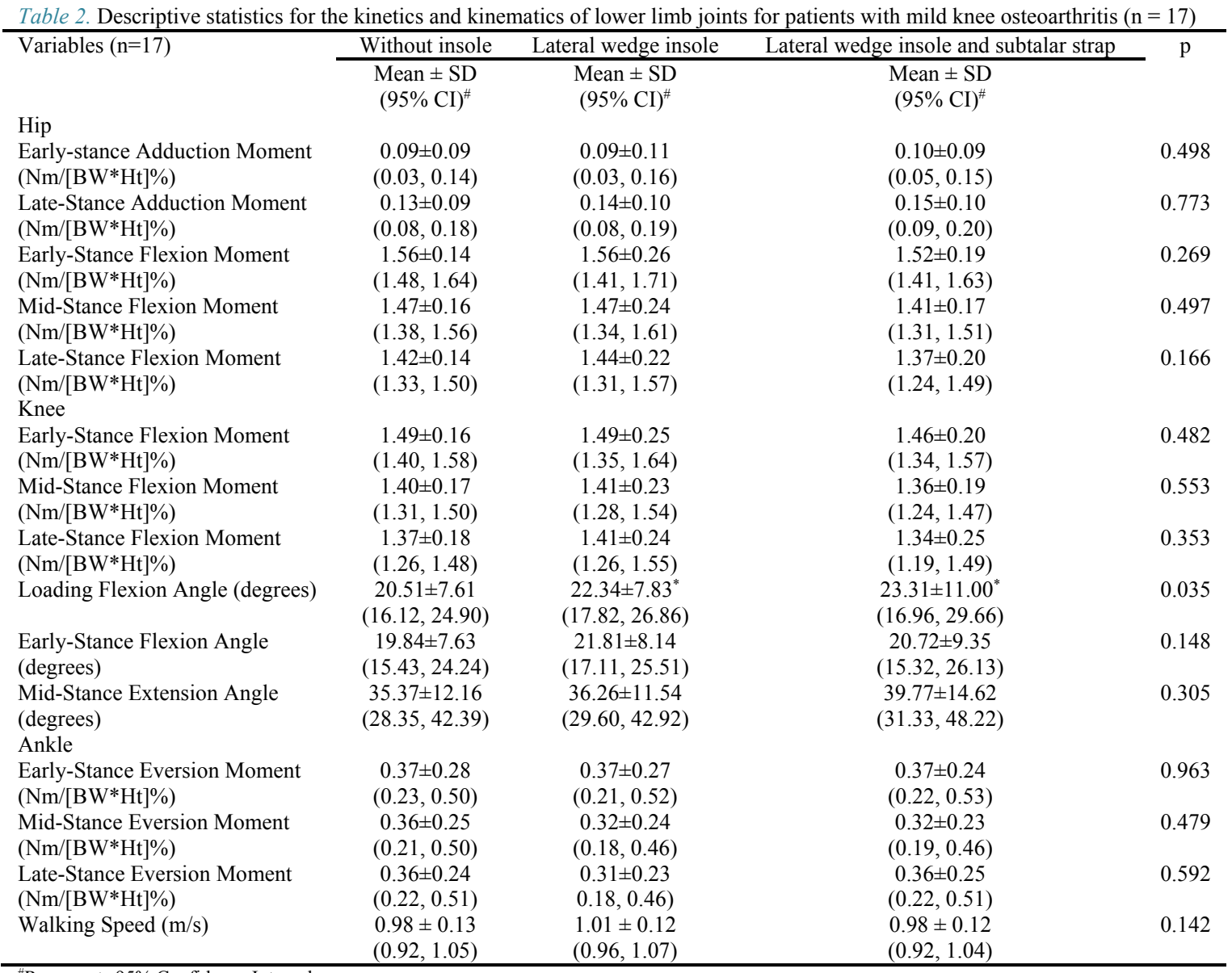

"Represents $95 \%$ Confidence Interval

*Statistically significant $(\mathrm{P}$ value $<0.05$ ) in pairwise comparison

support ended to a protracted controversy $(7,13,14,31)$. The possible explanations could be related to the variation among the sample of the studies or the interventions used in the studies. For example, LWI with a medial arch support could decrease EKAM in healthy participants (32). Healthy individuals were more adaptive than patients with KOA in this study. However, LWI and an arch support with $7^{\circ}$ lateral inclination could decrease the early and late peaks of EKAM in patients with KOA (10). Here, a $5^{\circ}$ wedge was used, because it has been shown that smaller amount of wedge is associated with foot comfort inside patients' shoes (25). Nevertheless, greater wedge could decrease EKAM in larger amounts compared to $5^{\circ}$ lateral inclination (25).

In this study, it was hypothesized that the subtalar strap could strengthen the effect of LWI (12, 33). However, according to the findings of a randomized controlled trial (34), using a subtalar strap with LWI was not superior to using a subtalar strap with neutral insole on pain and physical function in KOA (34). One reason may be the arch support used in our insoles, as arch support tends to provide a COP medial shift and counteracts the lateral shift of the COP made by the wedge (31). However, customized arch support in LWI can improve pain, physical function, and foot function after 2 months (13) and EKAM in patients with KOA (31). The promising effect of LWI with an arch support with soft ethyl-vinyl acetate was also shown on pain and reduced risk of falls in patients with mild KOA compared to baseline (14). Therefore, LWI with an optimal arch support may be beneficial to patients with KOA. In this study, the height of the arch support was high.

This study showed that LWI and a subtalar strap could increase the knee flexion angle at the loading phase compared to LWI. Here, it was hypothesized that using a subtalar strap with LWI could change the kinetic and kinematics of the lower limb joints, as limiting the ankle motion in the sagittal plane by using wedge could decrease the knee flexion angle at the early stance and affect the joints' range of motion (35). The increase of knee flexion angle at loading of the stance phase by using subtalar strap may show that LWI and a subtalar strap can affect the knee more than the hip joint. The quadriceps activity, responsible for the knee flexion angle and reduced in patients with KOA, may cause an increase in knee flexion angle at the loading (36), because the increase of knee flexion angle at the loading may not require the greater activation of the quadriceps. However, measuring the activity of the lower limb muscles was out of the scope of this study. Therefore, further studies should be conducted to assess the changes imposed by LWI and a subtalar strap on muscles activities. Despite all controversies among the 
effects of LWIs in KOA (7), implementing an early intervention is highly important to decrease the possibility of KOA progression (37). A 6-year follow-up on patients with KOA showed that $1 \%$ increase in EKAM caused 6.46 times increase in the risk of KOA progression (38).

This study had several limitations. First, the sample was limited to females. However, the authors believed that the prevalence of knee osteoarthritis is higher in females, especially those younger than 55 years $(2,39)$. The results of this study cannot be generalized to males and to other group of patients with more severe KOA. Nevertheless, the aim of this study was to assess the biomechanical effect of LWI on kinetics and kinematics of lower limb joints. Therefore, longitudinal clinical trials are highly recommended to assess the long-term effects of LWI and a subtalar strap for the effect of time on gait parameters.

\section{Conclusion}

In this study, no effect of LWI and concomitant use of a subtalar strap with LWI was found on loading of the medial compartment of the knee and lower limb joints in patients with mild KOA. Furthermore, the results of this study suggested that the potential effect of customized approaches to LWIs for KOA needs further consideration and may be identified as effective treatments for patients with KOA.

\section{Acknowledgements}

This study was funded by the research committee of Iran University of Medical Sciences.

This study was registered in IRCT (Iranian Registry of Clinical Trials) (IRCT201104185520N4).

\section{Conflict of Interests}

The authors declare that they have no competing interests.

\section{References}

1. Cross M, Smith E, Hoy D, Nolte S, Ackerman I, Fransen M, et al. The global burden of hip and knee osteoarthritis: estimates from the Global Burden of Disease 2010 study. Ann Rheum Dis. 2014;73(7):1323-30.

2. O'Connor MI. Osteoarthritis of the Hip and Knee: Sex and Gender Differences. Orthopedic Clinics of North America. 2006;37(4):55968.

3. Bennell KL, Bowles KA, Wang Y, Cicuttini F, Davies-Tuck M, Hinman RS. Higher dynamic medial knee load predicts greater cartilage loss over 12 months in medial knee osteoarthritis. Ann Rheum Dis. 2011;70(10):1770-4.

4. Creaby M, Wang Y, Bennell K, Hinman R, Metcalf B, Bowles K-A, et al. Dynamic knee loading is related to cartilage defects and tibial plateau bone area in medial knee osteoarthritis. Osteoarthr Cartilage. 2010;18(11):1380-5.

5. McAlindon TE, Bannuru RR, Sullivan MC, Arden NK, Berenbaum F, Bierma-Zeinstra SM, et al. OARSI guidelines for the non-surgical management of knee osteoarthritis. Osteoarthr Cartilage. 2014;22(3):363-88.

6. Esfandiari E, Kamyab M, Yazdi HR, Foroughi N, Sanjari MA. The Immediate Effect of Lateral Wedge Insoles, With and Without a Subtalar Strap, on the Lateral Trunk Lean Motion in Patients With Knee Osteoarthritis. Geriatr Orthop Surg Rehabil. 2013;4(4):127-32.

7. Shaw KE, Charlton JM, Perry CKL, de Vries CM, Redekopp MJ, White JA, et al. The effects of shoe-worn insoles on gait biomechanics in people with knee osteoarthritis: a systematic review and meta-analysis. Br J Sports Med. 2018;52(4):238-53.
8. Felson DT, Carter SC, Liu A, Parkes MJ, Callaghan MJ, Jones RK. The efficacy of lateral wedge insoles for painful medial knee osteoarthritis after patient screening: a randomised trial. Osteoarthr Cartilage. 2018;26:S17.

9. Khan MT. A comparative study of the effects of lateral wedge insole shoe modification on the patients with knee osteoarthritis. Osteoarthr Cartilage. 2018;26:S328.

10. Yeh HC, Chen LF, Hsu WC, Lu TW, Hsieh LF, Chen HL. Immediate Efficacy of Laterally Wedged Insoles With Arch Support on Walking in Persons With Bilateral Medial Knee Osteoarthritis. Arch Phys Med Rehabil. 2014;95(12):2420-7.

11. Toda Y, Tsukimura N. A 2-year follow-up of a study to compare the efficacy of lateral wedged insoles with subtalar strapping and inshoe lateral wedged insoles in patients with varus deformity osteoarthritis of the knee. Osteoarthr Cartilage. 2006;14(3):231-7.

12. Kuroyanagi Y, Nagura T, Matsumoto H, Otani T, Suda Y, Nakamura T, et al. The lateral wedged insole with subtalar strapping significantly reduces dynamic knee load in the medial compartment: Gait analysis on patients with medial knee osteoarthritis. Osteoarthr Cartilage. 2007;15(8):932-6.

13. Hunt MA, Takacs J, Krowchuk NM, Hatfield GL, Hinman RS, Chang R. Lateral wedges with and without custom arch support for people with medial knee osteoarthritis and pronated feet: an exploratory randomized crossover study. Foot Ankle Surg. 2017;10(1):20.

14. Esfandiari E, Sanjari MA, Jamshidi AA, Kamyab M, Yazdi HR Knee osteoarthritis at the early stage: The four-week effect of lateral wedge insole on pain and risk of falls. Med J Islam Repub Iran. 2018;32(1):94-9.

15. Mündermann A, Dyrby CO, Hurwitz DE, Sharma L, Andriacchi TP. Potential strategies to reduce medial compartment loading in patients with knee osteoarthritis of varying severity: Reduced walking speed. Arthritis Rheum. 2004;50(4):1172-8.

16. Mündermann A, Dyrby CO, Andriacchi TP. Secondary gait changes in patients with medial compartment knee osteoarthritis: Increased load at the ankle, knee, and hip during walking. Arthritis Rheum. 2005;52(9):2835-44.

17. Thorp LE, Sumner DR, Block JA, Moisio KC, Shott S, Wimmer MA. Knee joint loading differs in individuals with mild compared with moderate medial knee osteoarthritis. Arthritis Rheum. 2006;54(12):3842-9.

18. Kellgren JH, Lawrence JS. Radiological assessment of osteoarthrosis. Ann Rheum Dis. 1957;16(4):494-502.

19. Des Jarlais DC, Lyles C, Crepaz N, Group T. Improving the reporting quality of nonrandomized evaluations of behavioral and public health interventions: the TREND statement. Am J Public Health. 2004;94(3):361-6.

20. Guo Y, Logan HL, Glueck DH, Muller KE. Selecting a sample size for studies with repeated measures. BMC Med Res Methodol. 2013;13:100-

21. Faul F, Erdfelder E, Lang AG, Buchner A. G*Power 3: A flexible statistical power analysis program for the social, behavioral, and biomedical sciences. Behav Res Methods. 2007;39(2):175-91.

22. Altman R, Asch E, Bloch D, Bole G, Borenstein D, Brandt K, et al. Development of criteria for the classification and reporting of osteoarthritis: classification of osteoarthritis of the knee. Arthritis Rheum. 1986;29(8):1039-49.

23. Hunter DJ, Lo GH, Gale D, Grainger AJ, Guermazi A, Conaghan PG. The reliability of a new scoring system for knee osteoarthritis MRI and the validity of bone marrow lesion assessment: BLOKS (Boston-Leeds Osteoarthritis Knee Score). Ann Rheum Dis. 2008;67(2):206-11.

24. Hinman RS, Payne C, Metcalf BR, Wrigley TV, Bennell KL. Lateral wedges in knee osteoarthritis: What are their immediate clinical and biomechanical effects and can these predict a threemonth clinical outcome? Arthritis Care Res. 2008;59(3):408-15.

25. Kerrigan DC, Lelas JL, Goggins J, Merriman GJ, Kaplan RJ, Felson DT. Effectiveness of a lateral-wedge insole on knee varus torque in patients with knee osteoarthritis. Arch Phys Med Rehabil. 2002;83(7):889-93.

26. Toda Y, Tsukimura N. Influence of concomitant heeled footwear when wearing a lateral wedged insole for medial compartment osteoarthritis of the knee. Osteoarthr Cartilage. 2008;16(2):244-53.

27. Maly MR, Culham EG, Costigan PA. Static and dynamic biomechanics of foot orthoses in people with medial compartment 
knee osteoarthritis. Clin Biomech. 2002;17(8):603-10.

28. Hinman RS, Payne C, Metcalf BR, Wrigley TV, Bennell KL. Lateral wedges in knee osteoarthritis: What are their immediate clinical and biomechanical effects and can these predict a threemonth clinical outcome? Arthritis Care Res. 2008;59(3):408-15.

29. Hinman RS, Bowles KA, Bennell KL. Laterally wedged insoles in knee osteoarthritis: do biomechanical effects decline after one month of wear? BMC Musculoskelet. 2009;10(1):146.

30. Heiden TL, Lloyd DG, Ackland TR. Knee joint kinematics, kinetics and muscle co-contraction in knee osteoarthritis patient gait. Clin biomech. 2009;24(10):833-41.

31. Dessery Y, Belzile É, Turmel S, Corbeil P. Effects of foot orthoses with medial arch support and lateral wedge on knee adduction moment in patients with medial knee osteoarthritis. Prosthet Orthot Int. 2017;41(4):356-63.

32. Nakajima K, Kakihana W, Nakagawa T, Mitomi H, Hikita A, Suzuki R, et al. Addition of an arch support improves the biomechanical effect of a laterally wedged insole. Gait Posture. 2009;29(2):208-13

33. Esfandiari E, Kamyab M, Foroughi N, Yazdi HR. The effect of lateral wedge insole with and without sub-talar strap on pain, function, and quality of life in medial knee osteoarthritis. Mod Rehabil. 2012;6(1).

34. Campos GCd, Rezende MU, Pasqualin T, Frucchi R, Bolliger Neto R. Lateral wedge insole for knee osteoarthritis: randomized clinical trial. Sao Paulo Med J. 2015;133:13-9.

35. Macrum E, Bell DR, Boling M, Lewek M, Padua D. Effect of limiting ankle-dorsiflexion range of motion on lower extremity kinematics and muscle-activation patterns during a squat. J Sport Rehabil. 2012;21(2):144-50.

36. Hurley MV, Scott DL, Rees J, Newham DJ. Sensorimotor changes and functional performance in patients with knee osteoarthritis. Ann Rheum Dis. 1997;56(11):641-8.

37. Ding C, Jones G, Wluka AE, Cicuttini F. What can we learn about osteoarthritis by studying a healthy person against a person with early onset of disease? Curr Opin Rheumatol. 2010;22(5):520-7.

38. Miyazaki T, Wada M, Kawahara H, Sato M, Baba H, Shimada S. Dynamic load at baseline can predict radiographic disease progression in medial compartment knee osteoarthritis. Ann Rheum Dis. 2002;61(7):617-22.

39. Cho HJ, Chang CB, Kim KW, Park JH, Yoo JH, Koh IJ, et al. Gender and Prevalence of Knee Osteoarthritis Types in Elderly Koreans. J Arthroplasty. 2011;26(7):994-9. 